\title{
A LARGE APERTURE SPECTROMETER AT FERMILAB TO STUDY HIGH MASS DIMUONS
}

\author{
E. ANASSONTZIS, S. KATSANEVAS, P. KOSTARAKIS, C. KOURKOUMELIS, A. MARKOU, \\ L.K. RESVANIS and G. VOULGARIS
}

University of Athens, Athens, Greece

\author{
M. BINKLEY, B. COX, J. ENAGONIO, G. HALE, C. HOJVAT, D. JUDD, K. KEPHART, \\ R.D. KEPHART, P.K. MALHOTRA, P.O. MAZUR, C.T. MURPHY, F. TURKOT, \\ R.L. WAGNER, D. WAGONER and W. YANG \\ Fermi National Accelerator Laboratory, Batavia, Illinois 60510, USA
}

\author{
H. ARETI, S. CONETTI, P. LEBRUN, D.G. RYAN, T. RYAN, W. SCHAPPERT and D.G. STAIRS \\ McGill University, Montreal, Quebec H3A 2T8, Canada
}

C. AKERLOF, X. CUI, P. KRAUSHAAR, D. NITZ, R. THUN and L. WANG

University of Michigan, Ann Arbor, Michigan 48109, USA

HE MAO and ZHANG NAI-JIAN

Shandong University, Jinan, Shandong, P.R. China

Received 18 March 1985 and in revised form 24 June 1985

\begin{abstract}
A large acceptance forward spectrometer located in a unique antiproton enriched beam has been used at Fermi National Accelerator Laboratory by Experiment 537 to study the production of high mass muon pairs. When the beam was operated at a momentum of $125 \mathrm{GeV} / c$, it had a flux of $1.5 \times 10^{7}$ particles per second of which $18 \%$ were antiprotons. The spectrometer was of closed geometry design and used drift chambers as the tracking elements. Operating over a relatively short period, the experiment accumulated the largest sample of antiproton-induced high mass dimuons of any experiment to date. The contamination of this data by pion-induced dimuons was less than $0.5 \%$.
\end{abstract}

\section{Introduction}

Experiment 537 has measured the production of high mass dimuons in $125 \mathrm{GeV} / c \overline{\mathrm{p}} \mathrm{N}$ and $\pi^{-} \mathrm{N}$ interactions using a large aperture forward spectrometer at Fermilab. The experiment was located in the High Intensity Laboratory of Proton West and used a beam which collected antiprotons from antilambda decays. Due to the small cross sections characterizing high mass dimuon production ( $\sim 10^{-9}$ of the total cross section), the experiment required a spectrometer of large acceptance that was capable of operating at high rates.

In three months of actual data taking, the experiment measured dimuons from $2 \times 10^{11} \overline{\mathrm{pN}}$ and $6 \times 10^{11}$ $\pi^{-} \mathrm{N}$ interactions. These interactions produced $4 \times 10^{6}$ triggers which yielded 40000 reconstructed $\psi$ 's and 2000 reconstructed continuum dimuons in the range $4<\mathrm{M}_{\mu \mu}<9 \mathrm{GeV} / c^{2}$.

\section{Beam}

The antiproton enriched beam [1] shown in fig. 1 was formed from decay products of the neutral secondaries produced by $400 \mathrm{GeV}$ primary protons interacting in a $30.5 \mathrm{~cm}$ long beryllium target. The neutral secondary beam was created by magnetically sweeping all charged particles out of the beam channel immediately downstream of the production target. Antiprotons and negative pions from antilambdas, lambdas, and neutral kaons decaying in this channel were collected by a quadrupole triplet. This negative flux was transported through a FODO channel and focused on the experiment target by a quadrupole triplet. The beam line was constructed from conventional dipole and quadrupole magnets and could operate up to a momentum of $300 \mathrm{GeV} / c$.

The beam consisted almost entirely of $\bar{p}$ and $\pi^{-}$ since no mechanisms exist which would produce a $\mathrm{K}^{-}$ 


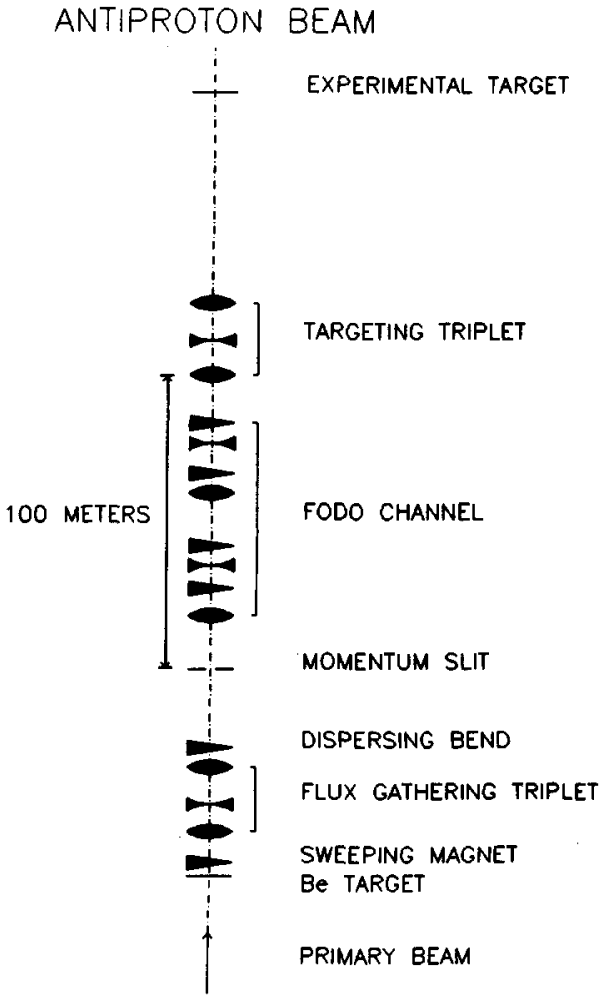

Fig. 1. Schematic layout of the tertiary antiprotein beam used for E-537. Toroidal iron spoilers that were used to suppress muon halo are not shown.

component. Any electron component was suppressed by a $2.5 \mathrm{~cm}$ thick lead absorber in the beam. At $125 \mathrm{GeV} / \mathrm{c}$ the average $\overline{\mathrm{p}} / \pi^{-}$ratio was 0.22 and the $\overline{\mathrm{p}}$ yield was approximately $2 \times 10^{6}$ particles per $6 \times 10^{12} 400 \mathrm{GeV}$ primary protons incident on the beryllium target. The $\overline{\mathrm{p}} / \pi^{-}$ratio is shown as a function of tertiary beam energy in fig. 2. Also shown in fig. 2 is the $\overline{\mathrm{p}} / \pi^{-}$ratio measured when the beam was operated as a standard charged beam with zero degree production. The momentum spread of the tertiary beam at $125 \mathrm{GeV} / c$ was $15 \mathrm{GeV} / c \mathrm{fwhm}$ and the angular divergence at the experimental target was $\pm 0.5 \mathrm{mrad}$. The momentum distribution of the beam and the $x$ and $y$ projections of the beam profile at the experimental target are shown in figs. 3(a), 3(b) and 3(c), respectively.

The beam tagging and momentum measuring systems are shown in fig. 4. Beam particles were identified as pions or antiprotons by two similar differential gas Cherenkov counters, $\mathrm{C} 1$ and $\mathrm{C} 2$ [2]. The upstream counter, $\mathrm{Cl}$, was used to identify antiprotons and the downstream counter, $\mathrm{C} 2$, was used to identify pions. These counters were sensitive to light emitted in cones with half-angles from 4.4 to $7.9 \mathrm{mrad}$ and 5.7 to $7.6 \mathrm{mrad}$, respectively. At $125 \mathrm{GeV} / c$ the operating pressures of

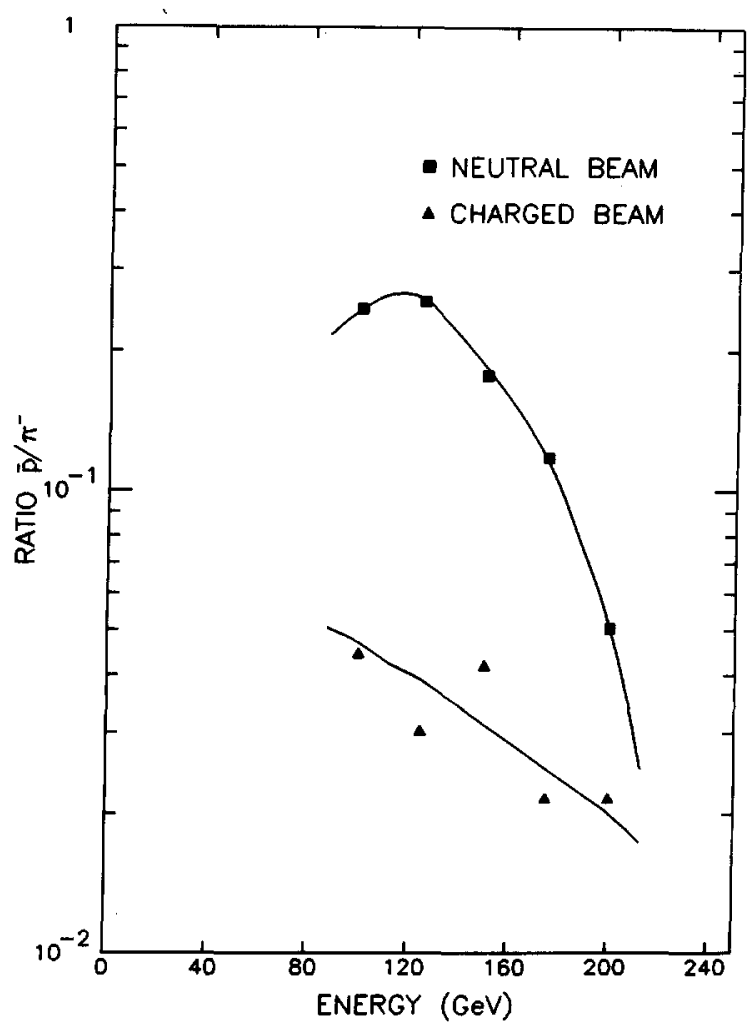

Fig. 2. Ratio of $\overline{\mathrm{p}}$ to $\pi^{-}$flux for the antiproton enriched beam. The lower curve is the corresponding ratio for a zero degree charged secondary beam.

the $80 \%$ helium/20\% nitrogen gas mixtures in $\mathrm{C} 1$ and $\mathrm{C} 2$ were 10.5 psia and 3.5 psia, respectively. A typical pressure curve is shown in fig. 5. RCA $31000 \mathrm{M}$ photomultipliers with transistorized bases [3] and LeCroy VV100B amplifiers permitted operation of the Cherenkov counters at beam intensities exceeding $3 \times 10^{7}$ particles/second. The average number of photoelectrons per beam particle ranged from 7-10 and the discriminators were set to trigger on $\geqslant 2$ photoelectrons. The $\overline{\mathbf{p}}$ Cherenkov counter, $\mathrm{C} 1$, operated at a pressure well above the threshold for pions, and approximately 5-10\% of the signals from this counter were actually produced by pions. This contamination of the antiproton data sample by misidentified pions was reduced to less than $0.5 \%$ by rejecting events for which a beam particle had been counted in both $\mathrm{C} 1$ and $\mathrm{C} 2$.

The trajectories of individual beam particles were measured by three beam monitoring stations positioned as shown in fig. 4. Each station included a proportional wire chamber. These wire chambers had three planes each consisting of 128 wires with $1.06 \mathrm{~mm}$ spacing. One plane had horizontal wires to measure the vertical coordinate of the beam. The other two planes in a given station were rotated by $\pm 60^{\circ}$ with respect to the hori- 

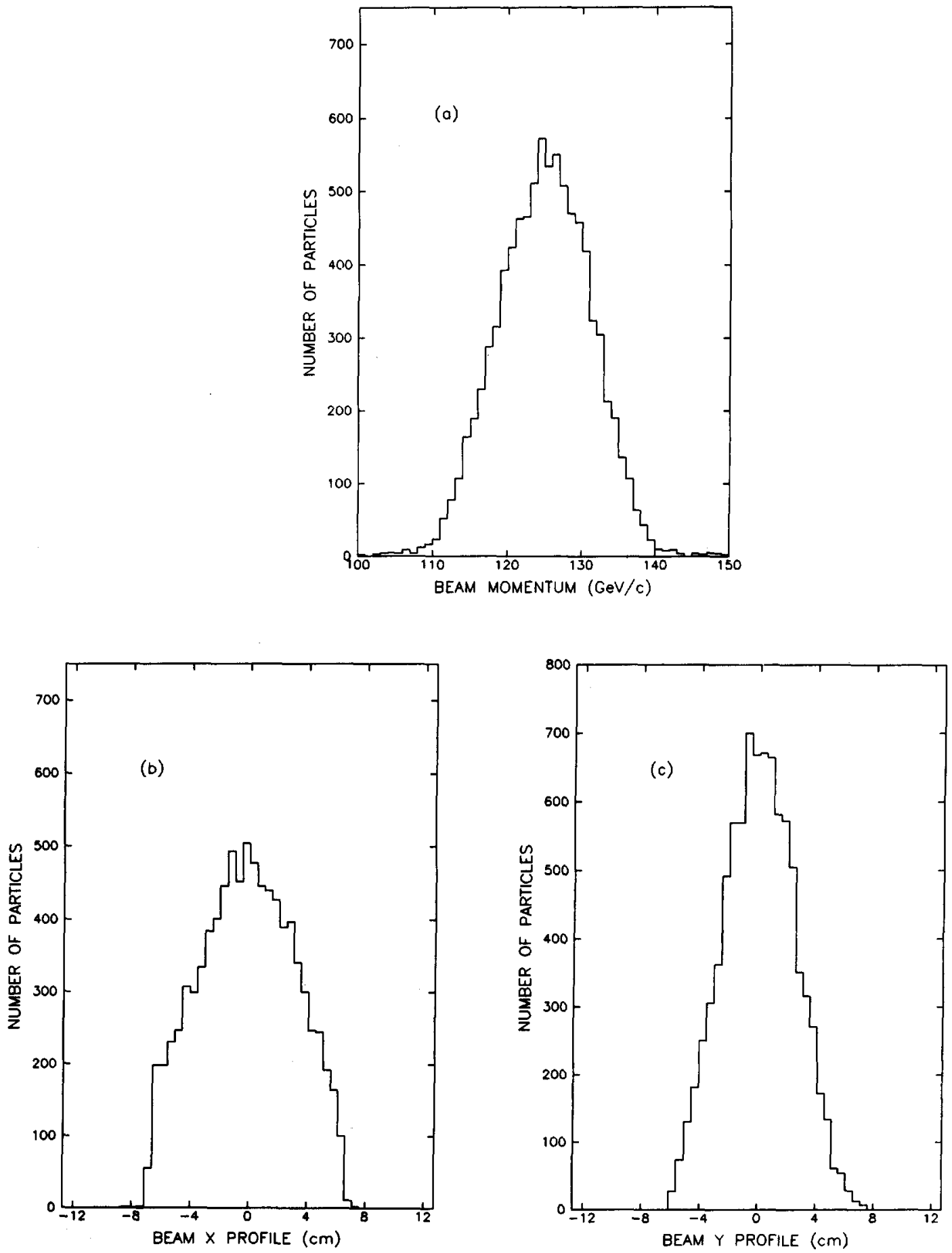

Fig. 3. Measured distributions of the antiproton beam: (a) momentum spread; (b) horizontal profile and (c) vertical profile at the experimental target.

zontal. The chambers used a gas mixture of $25 \%$ isobutane, 5\% methylal, and 0.5\% Freon 13B1 in argon. A discrete component amplifier [4] followed by a Fairchild $\mu$ A-685 comparator provided ECL differential "time over threshold" outputs from the beam chambers. The comparator output was transmitted on a custom T\&B 


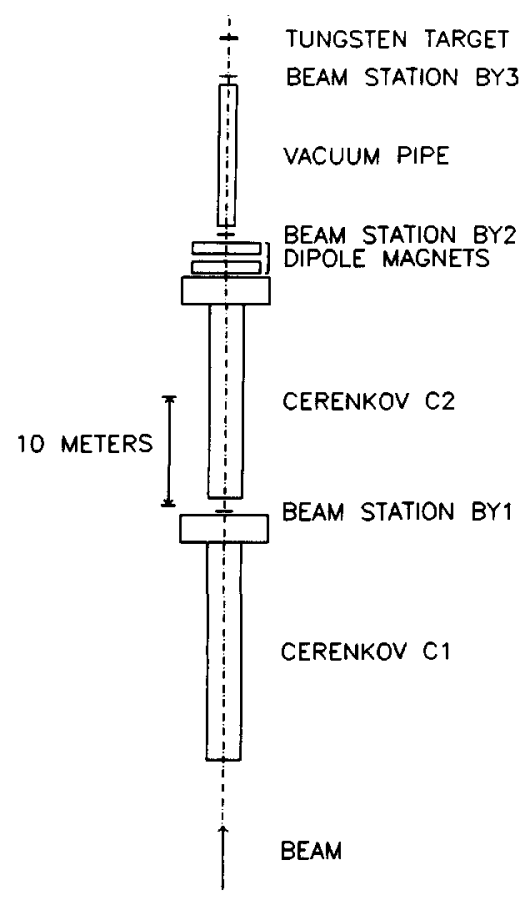

Fig. 4. Schematic layout of the beam tagging and momentum measuring system. The dipole magnets bend the beam in the vertical plane.

Ansley ribbon cable of up to $135 \mathrm{~m}$ in length to coincidence registers in the counting room. For cable lengths greater than $100 \mathrm{~m}$, an active ECL signal regenerator was required at an intermediate point. Immediately upsteam of the middle beam monitoring station, two small dipole magnets added a vertical momentum component of $0.319 \mathrm{GeV} / c$ to the beam. This bend and the coordinate measurements allowed a beam momentum determination to an accuracy of $\pm 1 \mathrm{GeV} / c$ at a mean value of $125 \mathrm{GeV} / c$.

In addition to the beam wire chambers each beam monitoring station had an eight element scintillation counter hodoscope (BYi) that was used in the trigger and for rejection of out-of-time tracks within the resolving time of the beam chambers. These hodoscopes measured the vertical coordinate only and could resolve consecutive beam buckets (the rf structure at Fermilab consists of 2 ns beam buckets separated by $19 \mathrm{~ns}$. The widths of the hodoscope elements were chosen to produce equal counting rates in all elements.

To reduce the contribution of beam halo (mostly muons) to the trigger, vertical (VX) and horizontal (VY) scintillation counter hodoscopes were positioned around the beam upstream of the experimental target. These counters were used to veto beam particles accompanied by a halo muon. The effect of this veto depended on the beam tune and typically $4 \%$ of the beam particles were

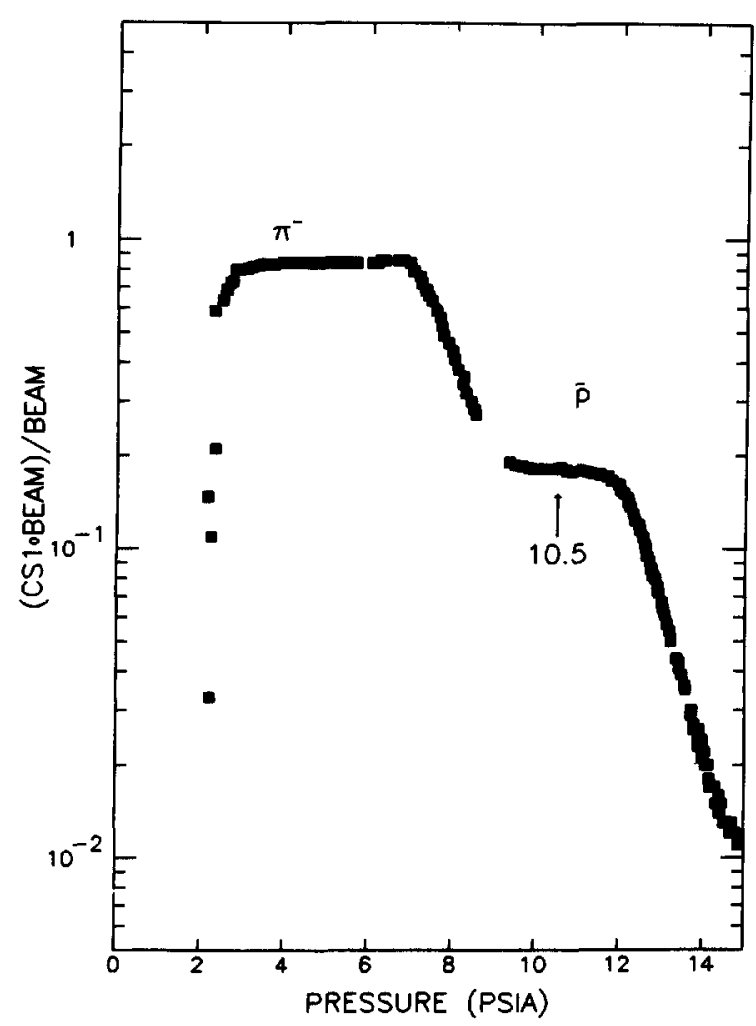

Fig. 5. Pressure curve for Cherenkov counter $\mathrm{C} 1$ indicating the operating point.

vetoed while the dimuon trigger rate was reduced by a factor of 4. In order to eliminate multiply occupied if buckets, beam particles were also vetoed if any 2 of the 3 beam hodoscope stations had more than one counter signal. "BEAM" was defined as a triple coincidence of the three beam stations (triple coincidence of the ORs of the eight elements composing each station) provided that neither the beam halo nor the two or more in an if bucket condition was present. A signal from Cherenkov counter $\mathrm{C} 1$ or $\mathrm{C} 2$ in coincidence with this BEAM signal defined $\mathrm{a} \overline{\mathrm{p}}$ or $\pi^{-}$particle provided $\mathrm{C} 1$ and $\mathrm{C} 2$ were not in coincidence. The logic diagram for the beam electronics is shown in fig. 6.

The $\overline{\mathrm{p}}$ and $\pi^{-}$beam signals were used in the trigger and also scaled for the absolute cross section determinations. The measured numbers of pions and antiprotons were corrected for multiply occupied rf buckets that were missed by the $\geqslant 2$ in a bucket veto due to the finite size of the hodoscope elements. This correction was typically $3 \%$ for pions and less than $0.5 \%$ for antiprotons. Antiprotons and pions in the same if bucket, as well as pion contamination of the signal from the antiproton Cherenkov counter, were effectively eliminated by rejecting triggers in which both $\mathrm{Cl}$ and C2 had counted. The Cherenkov counter efficiency was 


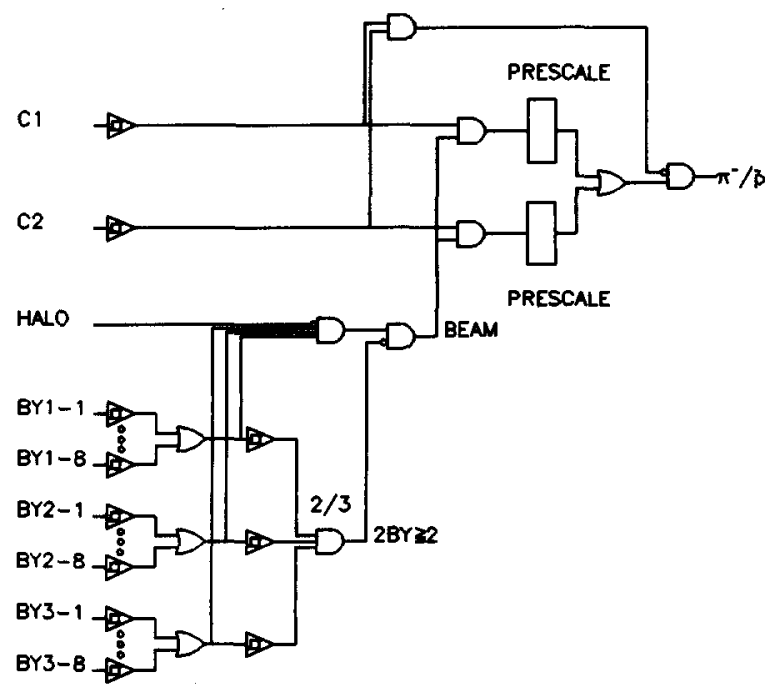

Fig. 6. Schematic logic diagram of the beam defining fast electronics.

continuously monitored by observing the quantity [ $\mathrm{Cl}$. BEAM + C2 - BEAM - (C1 · C2 $) \cdot$ BEAM $] / B E A M$, which was always greater than 0.993 .

\section{Spectrometer}

The spectrometer including target, hadron absorber, analysis magnet, proportional and drift chambers and muon detector is shown in fig. 7. The spectrometer's overall length was approximately $15.9 \mathrm{~m}$ and it had an angular acceptance for muons of $\pm 100 \mathrm{mrad}$ in the horizontal plane and $\pm 50 \mathrm{mrad}$ in the vertical plane. The individual elements of the spectrometer are now discussed in more detail.

\subsection{Targets}

Data were taken with $15 \mathrm{~cm}$ diameter segmented targets of tungsten, copper, and beryllium. These varied in thickness from 0.4 to 1.5 antiproton absorption lengths. Approximately $90 \%$ of the data was taken with the tungsten targets to maximize the rate for Drell-Yan events. The data from the beryllium and copper targets were used to measure the $A$ dependence of the $\psi$ cross section. Corrections were applied to the measured cross sections for the reinteraction of secondaries in the targets. Fig. 8 shows the relative cross sections as a function of tungsten target thickness for $\psi$ 's produced in $\pi^{-}$interactions, which constitute our largest individual data sample. Reinteraction predictions using the CASIM Monte Carlo code [5] were normalized to this result and then subsequently used to correct the smaller data samples of $\psi$ 's produced by antiprotons and continuum dimuons produced by both antiprotons and pions. A scintillation counter was positioned downstream of the target and additional counters were located between the various segments. The pulse heights in these counters were used to aid in the determination of the $z$ positions of the interactions. The distributions of the target segments along the beam direction were chosen to minimize any differences in acceptance between the various nuclear targets.

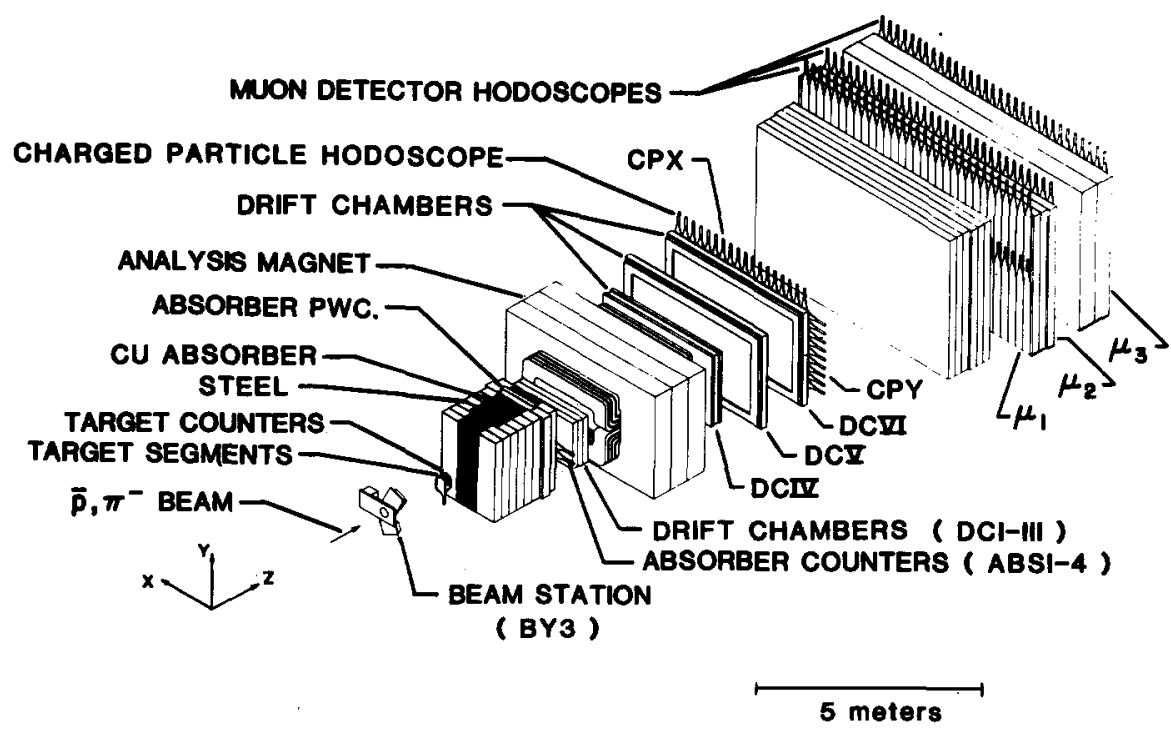

Fig. 7. General layout of the spectrometer used by E-537 to measure high mass dimuon production. The coordinate system used is indicated where the $y$ direction is vertical. 


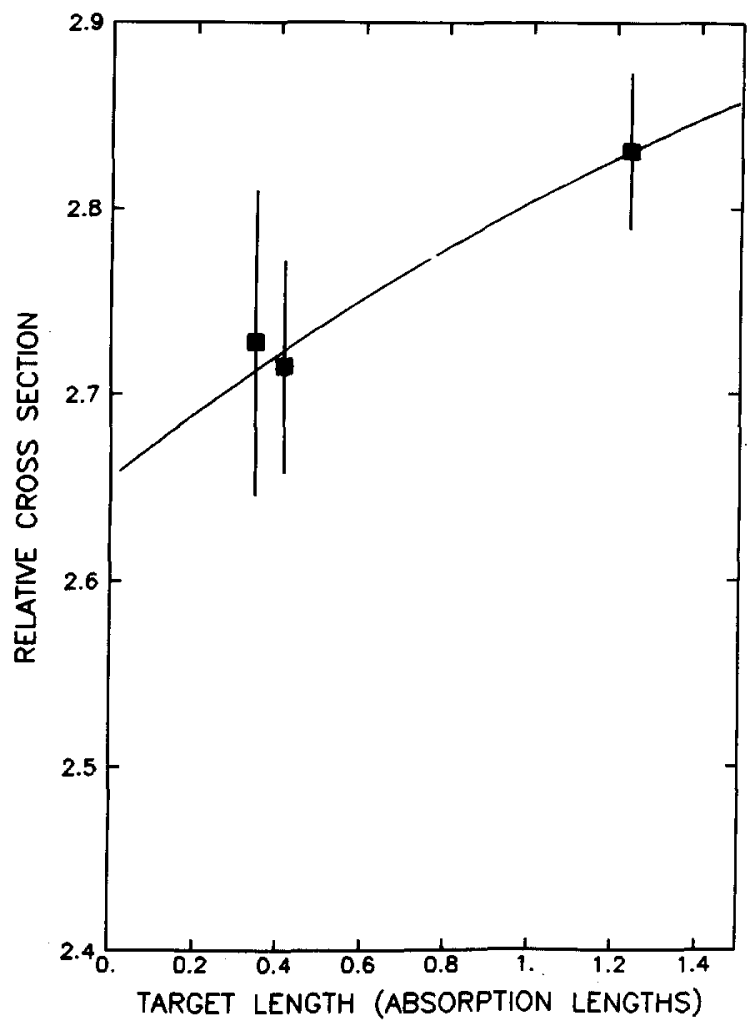

Fig. 8. Relative cross sections for $\psi$ production by $\pi^{-}$as a function of tungsten target thickness. The increase with target thickness is due to reinteraction.

\subsection{Hadron absorber}

A hadron absorber, which also served as a beam dump, was placed downstream of the target. The distance $(39 \mathrm{~cm})$ between the downstream end of the target and the absorber was made as small as possible consistent with allowing events originating in the target to be distinguished from those originating in the beam dump. Fig. 9 shows the $z$ coordinates of reconstructed dimuon vertices, demonstrating the clean separation of target produced events. The $1.5 \mathrm{~m}$ long (10.1 antiproton absorption lengths) copper absorber covered the full acceptance of the spectrometer. Copper was chosen because of its relatively high density, acceptable ratio of radiation length to absorption length and because it is non-magnetic. Steel was placed on both sides of the copper absorber to attenuate leakage from the sides and to degrade beam halo particles.

Imbedded in the hadron absorber at a distance of $1.25 \mathrm{~m}$ from the upstream end was a proportional wire chamber which consisted of $u$ and $v$ planes with wires oriented at $\pm 16.7^{\circ}$, respectively, with respect to the vertical direction. This absorber chamber had a $1.5 \mathrm{~mm}$ wire spacing. It measured the muon track coordinates as

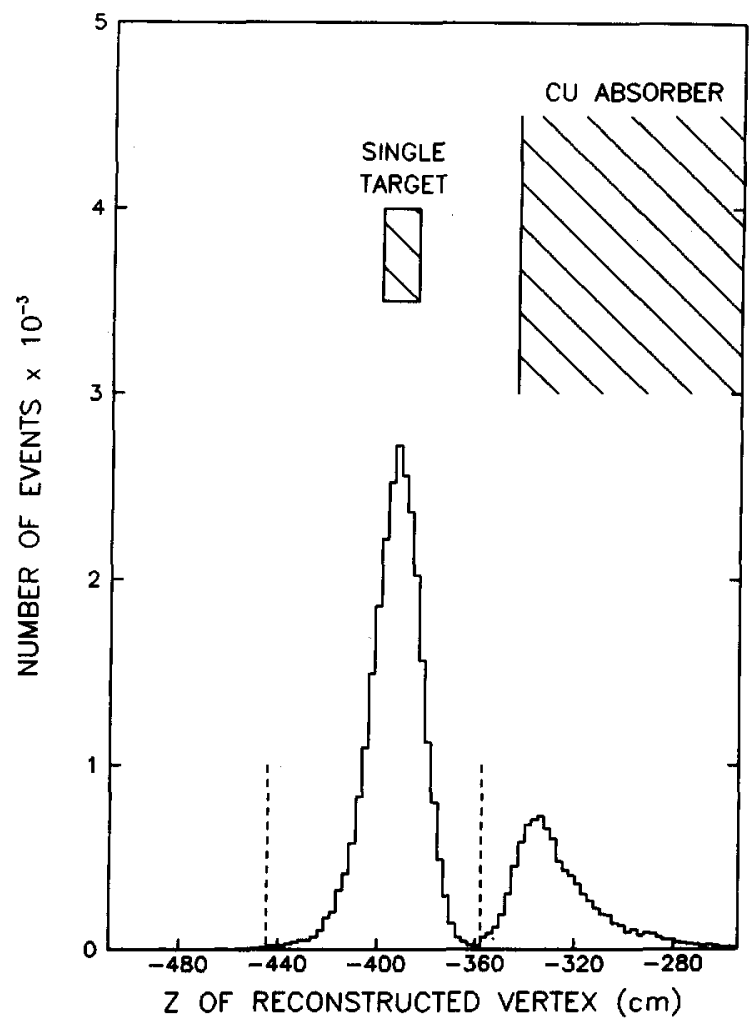

Fig. 9. Reconstructed dimuon vertex positions for the 1.5 absorption length tungsten target. The cuts used are indicated.

far upstream as possible to minimize the effect of multiple scattering in the copper absorber and thereby improved both the reconstructed $z$ position of the interaction vertex and the dimuon mass resolution.

\subsection{Analysis magnet}

The analysis magnet was a 150 ton window-frame dipole with saddle coils each containing 240 turns. The aperture was $91 \mathrm{~cm}$ high, $182 \mathrm{~cm}$ wide, and $152 \mathrm{~cm}$ long. At the nominal setting of $2400 \mathrm{~A}$, the main component of the field produced a horizontal momentum change of $830 \mathrm{MeV} / c$. The field distribution was mapped by charge induction in three orthogonal coils whose outputs and positions were recorded as they were moved through the field region. The absolute normalization of these data was established by measurements of the magnetic field using an NMR probe. The current and magnetic field in the analysis magnet were continuously monitored with a precision current shunt and a Hall probe attached to the lower pole face. The relative values of the current shunt and Hall probe readings always agreed within $0.1 \%$. Using the measured $\int B \cdot \mathrm{d} l$ of the magnet, the $\psi$ mass obtained by the experiment agreed with the accepted value within $1 \%$. 


\subsection{Drift chambers}

The spectrometer was equipped with six drift chambers to measure the trajectories of the charged particles. Each chamber had three signal planes and two of the cathode planes were shared. The wires in the $x$ plane were vertical to measure the horizontal coordinate; the wires in the $u$ and $v$ planes were oriented at the same angles $\left( \pm 16.7^{\circ}\right.$ to the vertical, respectively) as the $u$ and $v$ planes of the absorber proportional chamber.

Every second wire in each signal plane was a field shaping wire set at the same potential as the cathode planes. The spacing of wires in all signal planes was such that the cell cross section was square when projected in the $x-z$ plane. As a result, the perpendicular distance between wires in the $u$ and $v$ planes was less than in the $x$ plane by a factor of $\cos 16.7^{\circ}$. The cathode planes were constructed of either wires or stretched aluminium foil. In chambers using foil cathode planes, the cathode planes and field shaping wires were at ground potential, and the signal wires were run at positive high voltage and connected to the amplifiers using $200 \mathrm{pF}$ capacitors. In chambers using wire cathode planes, the cathode planes and field shaping wires were run at negative high voltage, and the signal wires were connected directly to the amplifiers. On the outside of the chambers with wire cathodes, it was necessary to supplement the multiwire ground plane with a thin foil ground plane to obtain adequate if shielding. The properties of each chamber are given in table 1.

A standard mixture of $50 \%$ argon and $50 \%$ ethane was used in all the drift chambers except for chamber $V$. Flow rates were typically set to change the chamber gas volume every $2 \mathrm{~d}$. Chamber $\mathrm{V}$ developed a constant discharge of $10 \mu \mathrm{A}$ which was eliminated by adding $0.8 \%$ ethanol to the argon/ethane mixture and raising
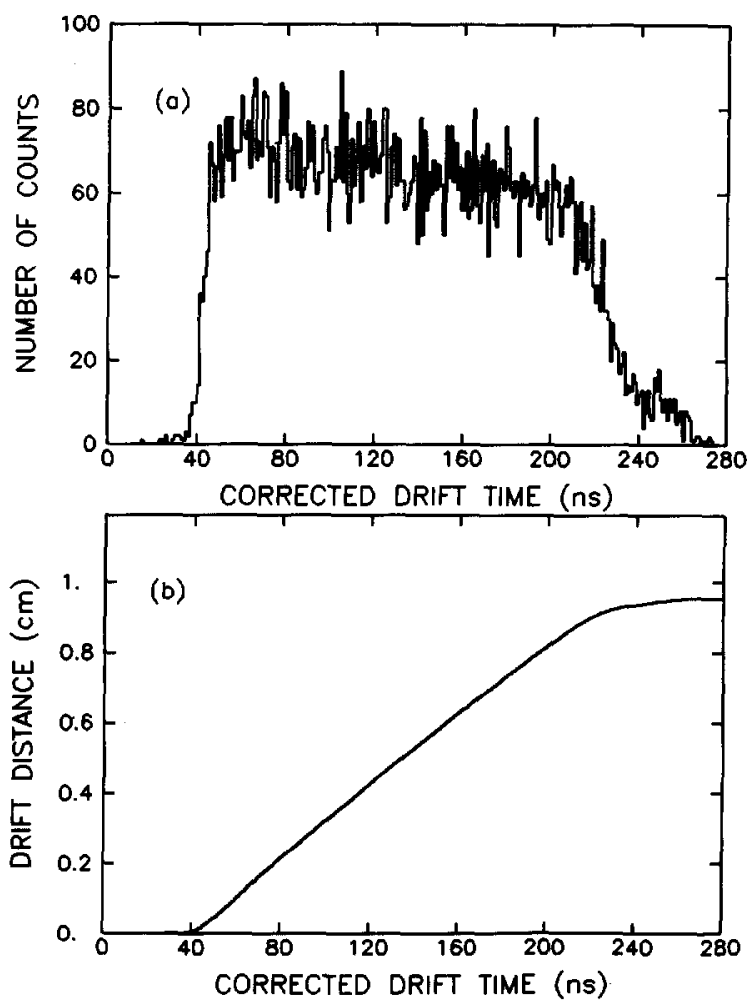

Fig. 10. (a) Drift times due to muon tracks in drift chamber IV. (b) The resulting relationship between drift time and drift distance.

the high voltage $2 \%$. The chamber readout electronics consisted of 16-channel LeCroy Model 7790 amplifier cards using Model MVL100 monolithic amplifier/discriminator chips. The discriminator thresholds were set

Table 1

Properties of the six drift chambers

\begin{tabular}{|c|c|c|c|c|c|}
\hline $\begin{array}{l}\text { Chamber } \\
\text { number a) }\end{array}$ & $\begin{array}{l}\text { Cell } \\
\text { size } \\
(\mathrm{mm})\end{array}$ & $\begin{array}{l}\text { Tungsten } \\
\text { signal wire } \\
(\mathrm{mm})\end{array}$ & $\begin{array}{l}\text { Chamber } \\
\text { cathode plane } \\
(\mathrm{mm})\end{array}$ & $\begin{array}{l}\text { CuBe field } \\
\text { shaping wire } \\
(\mathrm{mm})\end{array}$ & $\begin{array}{l}\text { Effective } \\
\text { aperture } \\
\left(\mathrm{m}^{2}\right)\end{array}$ \\
\hline I & 6 & 0.020 & $\begin{array}{r}0.0635 \text { diam. wire } \\
(1 \mathrm{~mm} \text { spacing })\end{array}$ & 0.0635 & $0.51 \times 1.02$ \\
\hline II & 12.7 & 0.025 & $0.025 \mathrm{Al}$ foil & 0.127 & $0.51 \times 1.02$ \\
\hline III & 12.7 & 0.025 & $0.025 \mathrm{Al}$ foil & 0.127 & $0.51 \times 1.02$ \\
\hline IV & 19.05 & 0.025 & $0.025 \mathrm{Al}$ foil & 0.127 & $1.02 \times 2.04$ \\
\hline $\mathbf{V}$ & 19.05 & 0.025 & $\begin{array}{l}0.0635 \text { diam. wire } \\
\quad(1.9 \mathrm{~mm} \text { spacing })\end{array}$ & 0,0635 & $1.68 \times 3.66$ \\
\hline VI & 19.05 & 0.025 & $\begin{array}{l}0.0635 \text { diam. wire } \\
\text { (1.9 mm spacing) }\end{array}$ & 0.0635 & $1.68 \times 3.66$ \\
\hline
\end{tabular}

a) Particular care was taken for chambers with wire cathode planes to insure that these planes had a good ac reference to the local chamber ground. For chambers V and VI, each $8 \mathrm{~cm}$ band of cathode plane wires was separately connected to ground with $100 \mathrm{pF}$ capacitors at both the top and bottom of the chamber. This established a well-defined signal return path and reduced cross talk between adjacent signal wires. 
at $800 \mu \mathrm{V}$ across the $200 \Omega$ amplifier input resistors. Differential ECL output signals of $300 \mathrm{~ns}$ width from the discriminators were transmitted over $70 \mathrm{~m}$ of $\mathrm{T} \& \mathrm{~B}$ Ansley ribbon cable to LeCroy Model 2770 time digitizers located in the counting room and operated in the common stop mode. The complete system including digitizers was calibrated by pulsing the field shaping wires in all signal planes.

Fig. 10(a) shows the drift times (after calibration) of hits associated with muon tracks from the $x$ plane of drift chamber IV. Assuming a uniform hit density, the relationship between drift time and drift distance in fig. 10(b) was obtained by fitting the integrated number of events in fig. 10 (a) as a function of time, normalizing the result to the total number of events, and multiplying by the maximum drift distance $(0.9525 \mathrm{~cm})$. The drift chamber system achieved a spatial resolution of between 200 and $300 \mu \mathrm{m}$ per plane with an average efficiency of 0.99 in the absence of electronic dead time.

\subsection{Scintillation counter hodoscopes}

Several scintillation counter hodoscopes were used to form the trigger, identify muons, and eliminate outof-time tracks. Immediately downstream of the copper absorber and upstream of the analysis magnet were located four "absorber" counters each covering the full acceptance of one quadrant of the apparatus except for a $15 \mathrm{~cm}$ radius hole centered on the beam. Here and in the remainder of the paper, "quadrant" refers to a quadrant of the transverse $(x, Y)$ coordinate system, whose origin is at the geometric center of the spectrometer and coincides with the projected beam line position. One of the fast triggers (TRIG2) required that at least one track pass outside the $15 \mathrm{~cm}$ hole and hit an absorber counter, thereby biasing the trigger against low mass muon pairs.

Two highly segmented planes of scintillation counters were located downstream of drift chamber VI. The upstream plane (CPX) consisted of 88 vertical counters $(3.8 \mathrm{~cm} \times 101.6 \mathrm{~cm})$ extending upward from beam height and 88 similar counters extending downward. Each electronic "element" of CPX consisted of two vertically opposite physical counters, whose photomultiplier signals were added before discrimination. The downstream plane (CPY) had 24 horizontal counters $(7.62 \mathrm{~cm} \times 203.2$ $\mathrm{cm}$ ) on each side of the beam. The central counters in both planes were retracted leaving a $15 \mathrm{~cm} \times 15 \mathrm{~cm}$ hole centered on the beam to eliminate hits from high energy halo particles and muons in the beam. Two or more CPX counters were required in the fast trigger, and the CPY counters measured the $y$ component of the opening angle of each dimuon candidate for use in the trigger processor algorithm.

The muon detector, which presented an additional 13.2 interaction lengths for hadrons, was located down- stream of the charged particle hodoscopes. Muons were identified by three planes of scintillation counters, $\mu 1$, $\mu 2$, and $\mu 3$, imbedded in 300 tons of steel and concrete. This detector consisted of $1.2 \mathrm{~m}$ of steel followed by the first plane, $\mu 1,0.6 \mathrm{~m}$ of steel followed by the second plane, $\mu 2$, and $0.9 \mathrm{~m}$ of concrete followed by the third plane, $\mu 3$. In passing through the detector muons lost approximately $3.6 \mathrm{GeV}$ of energy loss in the copper hadron absorber and the muon detector was approximately $6 \mathrm{GeV}$, which determined the minimum muon energy that could trigger the spectrometer.

The three muon hodoscope planes each consisted of upper and lower rows. The individual elements in $\mu 1$, $\mu 2$, and $\mu 3$ were vertical counters $20.3,22.9$, and 26.7 $\mathrm{cm}$ wide, respectively. In the first plane, $\mu 1$, there were 30 counters in each of the upper and lower rows with no overlap. Central counters in the $\mu 1$ plane were retracted leaving a $40 \mathrm{~cm} \times 40 \mathrm{~cm}$ hole centered on the beam to avoid muon halo. In subsequent planes there were 31 slightly overlapped counters in each row arranged such that every $\mu 1$ counter had two corresponding counters in $\mu 2$ and in $\mu 3$. The sizes of the counters in $\mu 2$ and $\mu 3$ were chosen such that $99 \%$ of the muons passing through $\mu 1$ were counted after allowing for multiple scattering.

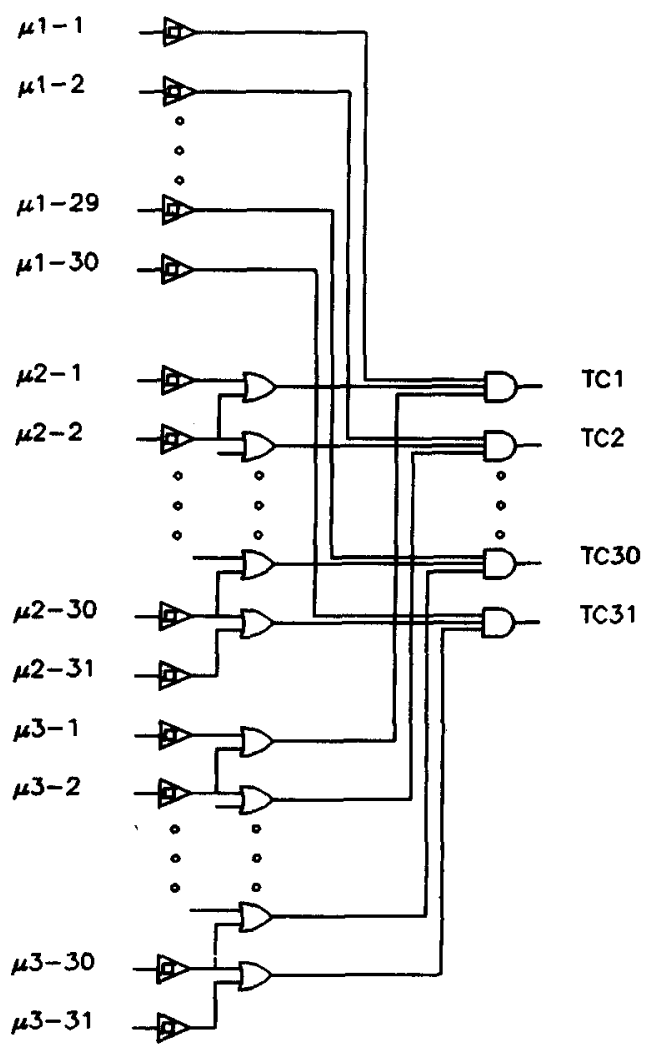

Fig. 11. Logic diagram for the triple coincidences between the three muon counter planes. 
The 60 counters in $\mu 1$ and their corresponding counters in $\mu 2$ and $\mu 3$ formed 60 triple coincidence trigger signals (TCi) used to generate the fast trigger and define roads for the trigger processor. We show in fig. 11 the logic circuitry used to produce the TCi signals. Special data runs were taken with only two of the three muon hodoscope planes required to form $\mathrm{TCi}$ in order to measure the muon counter efficiencies. Efficiencies were found to be 0.996 per counter.

\section{Fast trigger and trigger processor}

The fast trigger logic used for the experiment is shown in fig. 12, where three distinct trigger levels are indicated. The muon triple coincidences, TCi's, were first logically combined to form quadrants labeled HQ1-HQ4. The least restrictive trigger, TRIGO, was then formed if two or more separate muon quadrant signals were in time coincidence with either a $\bar{p}$ or $\pi^{-}$ beam particle. The next most restrictive trigger, TRIG1, was formed if two or more CPX counters had outputs in coincidence with TRIG0. Finally the most restrictive trigger, TRIG2, was formed by also requiring that at least one individual muon quadrant signal be coincident with the appropriate absorber counter quadrant ABS1-ABS4.

The fast triggers were formed in approximately 100 ns with the timing being determined by the BEAM

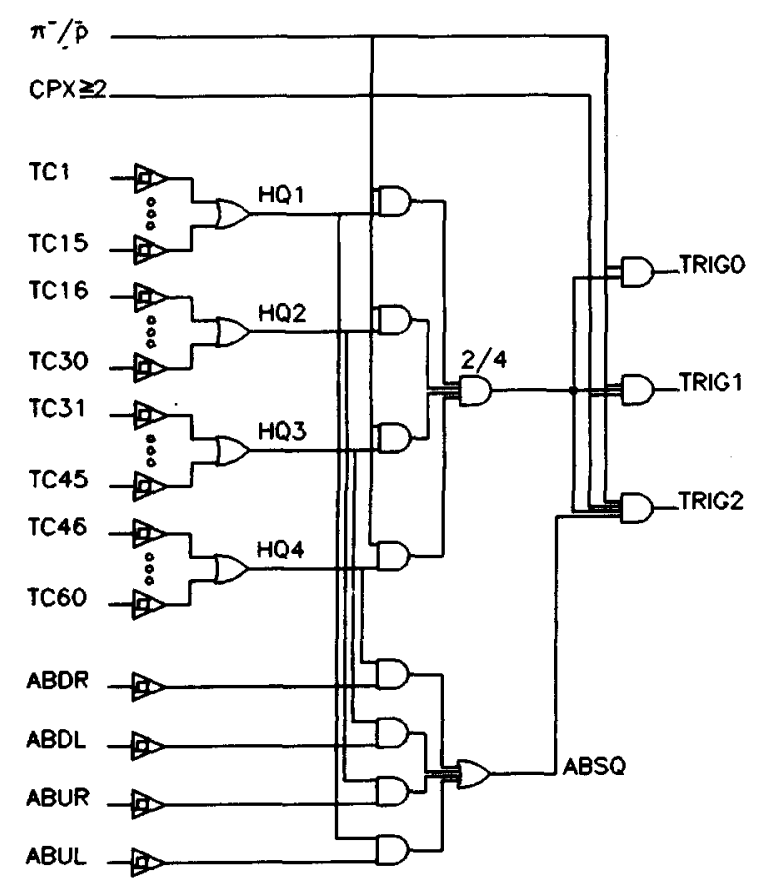

Fig. 12. Electronic logic used to define the fast triggers. Notation is defined in the text. signal. With the beam conditions described above the relative rates for TRIG0/TRIG1/TRIG2 were 5/2.5/1. The absolute rate depended on beam tune conditions; with good conditions the TRIG2 rate was approximately 400 per $10^{7}$ interactions in the target. The inefficiencies in TRIG1 and TRIG2 due to gaps between adjacent counters and individual counter efficiencies were $7 \%$ and $10 \%$ respectively. We accumulated most of the antiproton data using TRIGl and most of the pion data using TRIG2.

If an interaction satisfied the conditions for a fast trigger, information from trigger counters and the drift chamber system was routed to a fast trigger processor [6] employing ECL logic that was developed to further reduce the data rate. By matching hits in the $x$ planes of drift chambers IV, V, and VI with the CPX hodoscope counters, the processor found tracks and required that at least two of these point to muon counters which had formed the triple coincidences. If the correlation with the muon detector was positive, momenta of individual tracks were calculated by assuming that the interaction had occurred at the center of the target. The processor then calculated invariant masses for all pairs of tracks until a combination was found whose mass was greater than $2 \mathrm{GeV} / c^{2}$. The event was recorded if this minimum mass requirement was satisfied or if the event was sufficiently complex that the processor did not finish within $10 \mu \mathrm{s}$. For events that were rejected by the trigger processor, a fast clear was sent to the data acquisition electronics and a new trigger could be accepted after a total average deadtime of $6 \mu \mathrm{s}$. A factor of 5-10 reduction in the TRIG1 and TRIG2 rates was achieved with $99 \%$ efficiency for keeping good dimuon events with $M>2.5 \mathrm{GeV} / c^{2}$. The reduction in fast trigger rate for low mass values can be seen in fig. 13(a). The residual events with low masses are due either to events that passed the $2 \mathrm{GeV} / c^{2}$ cut due to the processor resolution or to events in which there was at least one other muon candidate that produced an accidental combination with invariant mass greater than $2 \mathrm{GeV} / c^{2}$. Fig. 13(b) shows the loss of events with masses greater than $2 \mathrm{GeV} / c^{2}$ due to trigger processor inefficiencies. More details about the operation of this trigger processor are given in ref. [6].

\section{Data acquisition system}

Data acquisition was controlled and monitored by a Digital Equipment Corporation PDP-11/45 computer with 128K words of memory using the RSX11-M operating system. The output of the trigger processor was used to interrupt the computer, which then read data from the experiment via three parallel CAMAC branch drivers using a Fermilab supported data acquisition program [7]. The drift chamber digitizers and propor- 

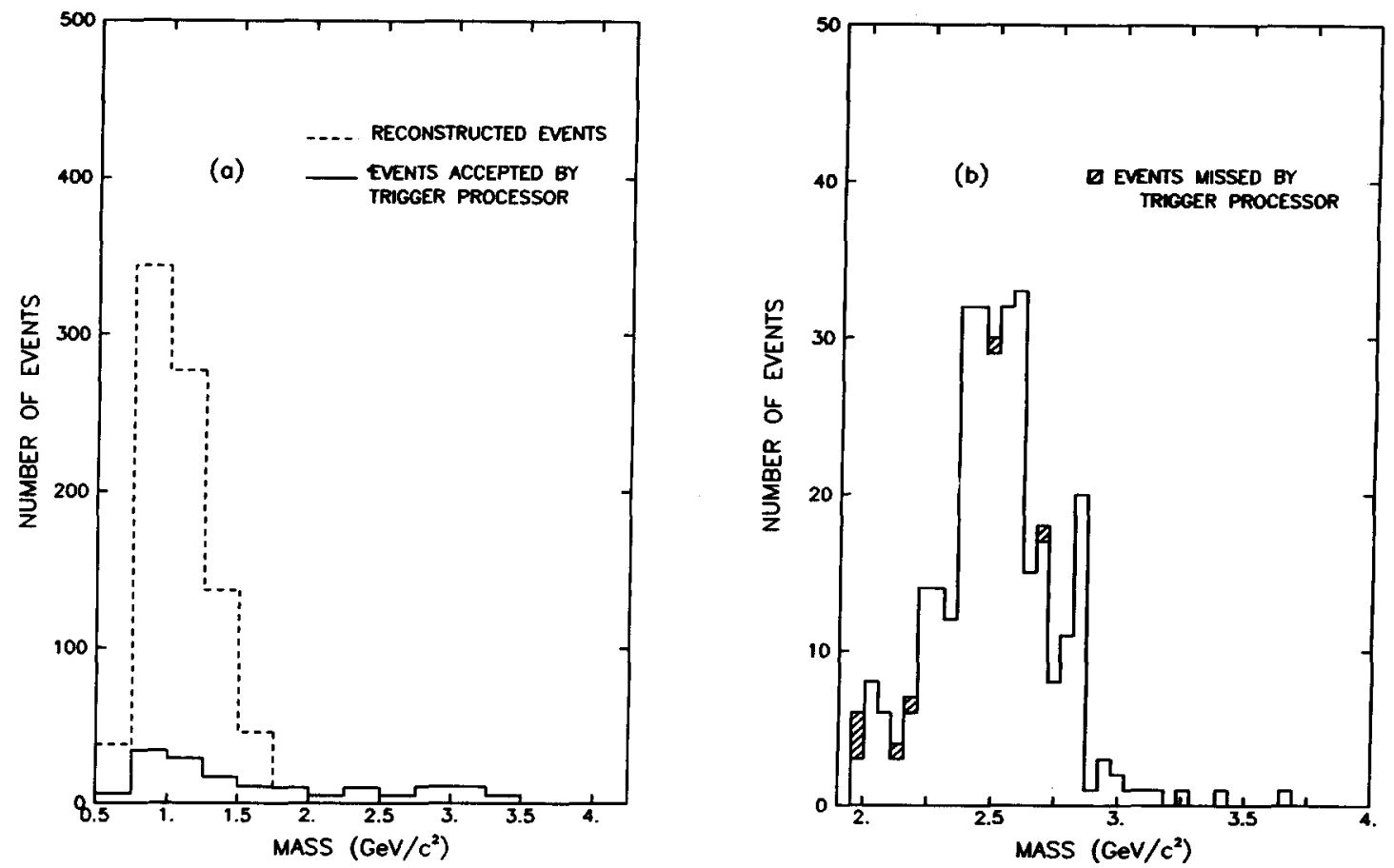

Fig. 13. (a) Events rejected by the trigger processor as a function of final reconstructed invariant mass. (b) Real events missed with the trigger processor threshold set at $2 \mathrm{GeV} / c^{2}$.

tional chamber latches were located on one branch and the various latches, ADCs, TDCs, and scalers for the other spectrometer elements required a second branch. The third brtanch was dedicated to the ECL trigger processor electronics.

A complete accelerator cycle at Fermilab varied in duration from 10 to $16 \mathrm{~s}$. During the one second long beam spill, data was stored in an additional $128 \mathrm{~K}$ words of bank-switched bulk memory and was written to tape between spills. The remaining time was used to process a sample of the events to verify that the apparatus was functioning properly. This was done with a versatile histograming and display system called SUPERGRAM [8]. By efficiencly using disk storage, SUPERGRAM was capable of accessing over $100 \mathrm{~K}$ bins of information while requiring only $6 \mathrm{~K}$ words of dedicated program memory space. It was therefore possible to simultaneously histogram all the quantities of interest in the experiment, with many used for more than one histogram. Sufiicient time was available to process $10 \%$ of the events in enough detail to insure that good data was being recorded.

\section{Event reconstruction}

The first step in event reconstruction was to form track segments downstream of the analysis magnet. The drift chamber hits were initially used without time information to reconstruct intersecting triplets of $x, u$, and $v$ wires for chambers IV to VI. These triplets were then combined to form rough track segments. When drift time information was added, the number of possible track segments increased due to the left-right position ambiguities. Only the subset of those track segments that satisfied restrictive minimum deviation cuts were kept and their $\chi^{2}$ values were calculated. These tracks were projected to the bend plane of the magnet and the bend plane points were then combined with all hits (including ambiguities) in the upstream chambers in order to form upstream track segments. A $\chi^{2}$ was calculated for the upstream segment and was added to ther downstream $\chi^{2}$ to give an overall total. The final selection of hits, allowing for ambiguities, was made by picking the solutions with the lowest total values of $\chi^{2}$. To eliminate track segments left in the chambers by particles from other interactions, all selected tracks were required to intersect with $\mathrm{CPX}$ counters and muon hodoscope counters that hasd contributed to the trigger. Using this technique, the efficiency for finding two tracks was $90 \%$ for high mass muon pairs. Most of the inefficiency was due to the discriminator dead time $(\sim 300 \mathrm{~ns})$ produced by tracks from interactions which had occurred in the copper absorber.

After track segments had been matched in the front and rear of the magnet, a horizontal bend plane at the 
magnetic center was assumed to determine the momentum. A small correction was made for horizontal bending due to field beyond those chambers located close to the magnet gap. Two vertical bend planes were included at $\pm 99 \mathrm{~cm}$ from the magnet center to allow for weak focusing effects due to the $z$ component of the field. These bends could be as large as $6 \%$ of the horizontal bending in extreme cases. The deflection due to the $x$ component of the field was neglected since it was very small over most of the acceptance. Using the measured resolution of the drift chambers and the measured field integral, our expected momentum resolution was $\Delta p / p$ $=0.004 p$. This resolution contributed a newgligible amount to the observed $\psi$ mass resolution, which was dominated by the error in the opening angie measurement due to multiple scattering in the target and hadron absorber. This error was minimized by including information from the absorber proportional chamber and the beam measuring system.

Events which had two muon track candidates whose calculated invariant mass was greater than $2.0 \mathrm{GeV} / c^{2}$ were subject to further analysis to better distinguish events originating in the target from those produced in the beam dump. For this the information from the drift chambers, the absorber MWPC, and the beam chamber were combined using an algorithm similar to that of ref.
[9]. The measurements of the positions and angles of the muons as they exited from the absorber and the positions of the muons as measured by the absorber MPWC were used to calculate a probability function which depended on the $z$ position of the event vertex and the initial angles of the muons before they entered ther absorber. The transverse location of the interaction vertex was constrained by the reconstructed beam track using the beam chamber system. The probability function included position measurement errors and multiple scattering in the absorber. It was maximized by varying the $z$ coordinate of the vertex and the initial muon angles. The vertex distribution resulting from this fitting procedure is shown in fig. 9 for the 1.5 absorption length tungsten target.

Requirements were also placed on the distance between the tracks at the reconstructed vertex and on the transverse position of the vertex to ensure that the two muons did originate from a common point and were not the result of an accidental coincidence between a beam halo particle and a muon from the decay of a hadron. The reconstructed vertex was constrained transversely to be within $9.144 \mathrm{~cm}$ in $x$ and $10.16 \mathrm{~cm}$ in $y$ of the nominal beam spot at the target. As indicated in fig. 9, the $z$ position of the vertex was required to between $-444.3 \mathrm{~cm}$ and $-358.14 \mathrm{~cm}$. These requirements were
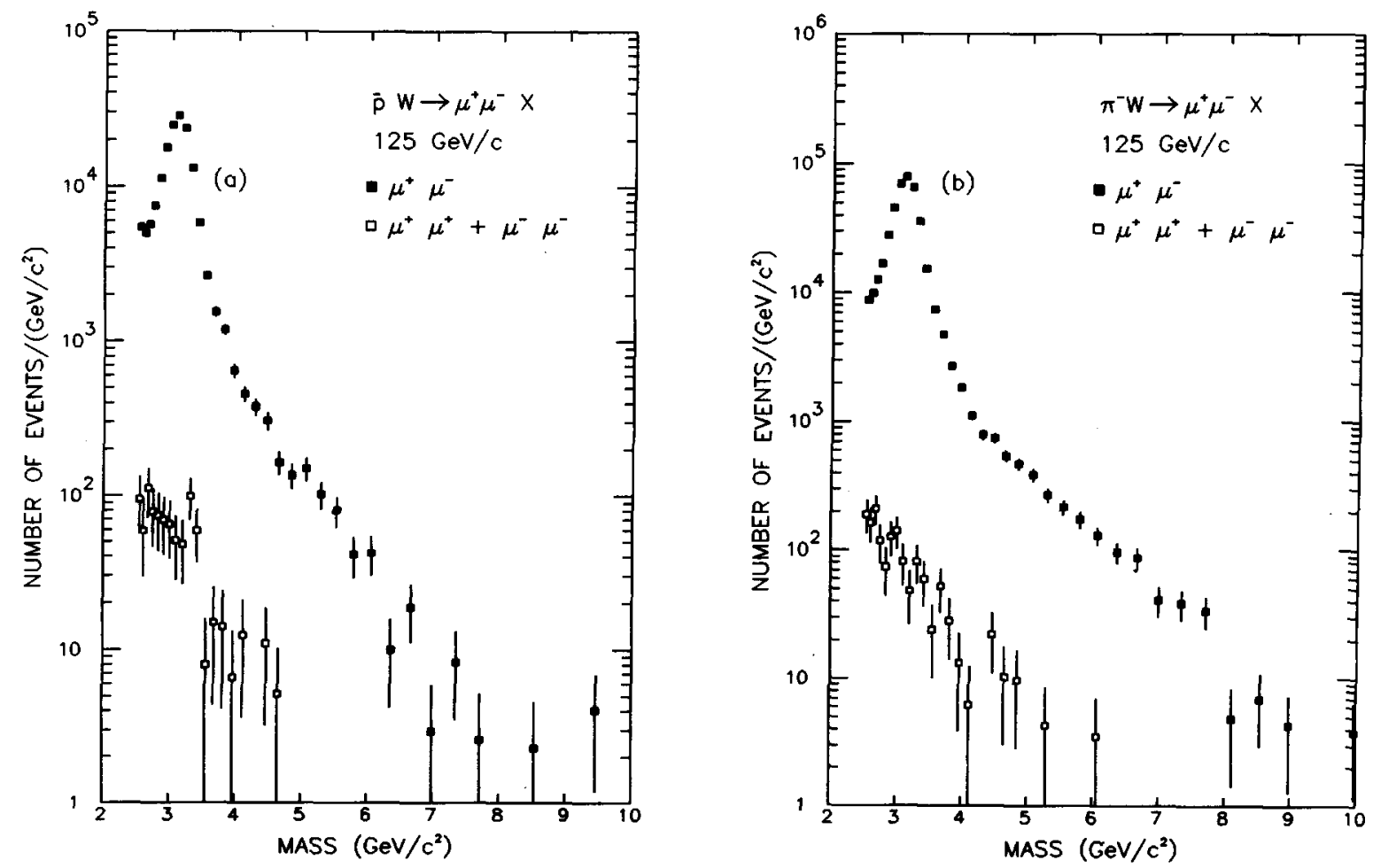

Fig. 14. Uncorrected dimuon mass spectrum produced by (a) $\overline{\mathrm{p}}$ and (b) $\pi^{-}$incident on a tungsten target at $125 \mathrm{GeV} / c$. The background level is shown by like sign pairs. The $\psi$ resonance is seen at $3.1 \mathrm{GeV} / c^{2}$. 
tested in the Monte Carlo simulation and resulted in a loss of less than $1 \%$ of the real events with no bias as a function of any of the kinematic variables.

Events with vertices in the target region were reanalyzed assuming that the $z$ position of the production vertex was at the centre of the target. This additional fitting procedure improved the $\psi$ mass resolution from $\sigma=270 \mathrm{MeV} / c^{2}$ to $185 \mathrm{MeV} / c^{2}$ for a 1.5 absorption length tungsten target. The comparable value for a 0.5 absorption length tungsten target was $140 \mathrm{MeV} / c^{2}$. The uncorrected mass spectra for both like and unlike charged muon pairs produced by $\overline{\mathrm{p}}$ and $\pi^{-}$interactions in the 1.5 absorption length tungsten target are shown in figs. 14(a) and 14(b) respectively.

\section{Acceptance}

The acceptance of the spectrometer was calculated using a Monte Carlo program to simulate both the geometric configuration and the electronic trigger cuts used. The results are shown as function of the Drell-Yan muon pair variables $M, x_{\mathrm{F}}, \rho_{\mathrm{T}}, \cos \theta$, and $\phi$ in fig. 15 for muon pairs with $4<M<9 \mathrm{GeV} / c^{2}$ produced by antiprotons. (Here $x_{F}$ is the ratio of the dimuon's longitudinal momentum to the maximum allowable momentum in the center of mass frame, $p_{\mathrm{T}}$ is the dimuon's transverse momentum, and $\theta$ and $\phi$ are the polar and azimuthal angles with respect to the beam direction of the positive muon in the dimuon's rest frame.) These acceptances include energy loss of the individual muons in the target, hadron absorber, and the muon identifier steel and concrete. Additional effects due to the configuration of the fast trigger, apparatus resolution, Fermi motion of the target nucleons, and track finding inefficiency were also included. The most prominent features of the acceptance curves are the decrease of acceptance for low values of $x_{F}$ and as $|\cos \theta| \rightarrow 1$. In both cases one of the muons is either swept away by the analysis magnet or has insufficient energy to penetrate to the last muon counter hodoscope. There is also some loss of dimuons with low invariant mass due to the hole left in the counter planes in the forward direction to avoid hits from beam punch through and from halo muons in and near the beam. The acceptance of the spectrometer averages $22 \%$ and is quite uniform as a function of the variables $p_{\mathrm{T}}, M$, and $\phi$.

\section{Summary}

We have described a beam and spectrometer used for the study of high mass dimuons produced in $\overline{\mathrm{p}} \mathrm{N}$ and $\pi^{-} \mathrm{N}$ interactions. The relatively high fraction (18\%) of antiprotons in the tertiary beam allowed us to study the antiproton induced reaction with higher statistics and
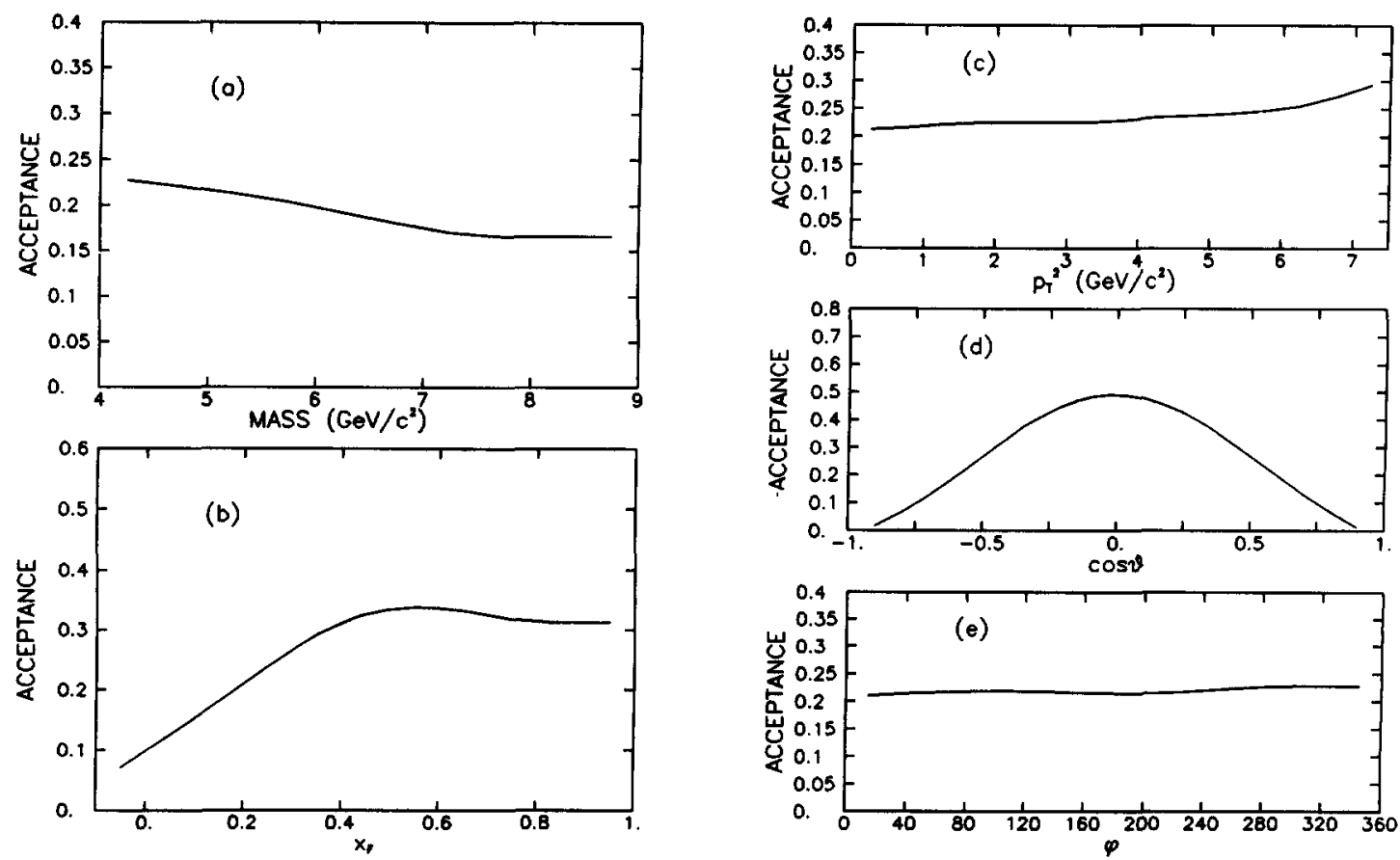

Fig. 15. Acceptances of the E-537 spectrometer as a function of the muon pair kinematic variables (a) $M$, (b) $x_{\mathrm{F}}$, (c) $p_{\mathrm{T}}^{2}$, (d) $\cos \theta$, and (e) $\phi$. 
smaller systematic errors than was possible in previouos experiments. Pions and antiprotons in the incident beam were identified by two differential Cherenkov counters, and their momenta were determined using MWPCs and two dipole magnets. The spectrometer was a closed geometry design consisting of a copper hadron absorber, drift chambers surrounding a large aperture analysis magnet to measure muon trajectory and momentum, and a set of scintillation hodoscope planes imbedded in 300 tons of steel to form a fast dimuon trigger. The fast trigger was complemented by an online trigger processor which used drift chamber wire information to compute invariant masses of candidate muon pairs and rejected events with masses below $2.0 \mathrm{GeV} / c^{2}$ in less than 10 $\mu \mathrm{s}$. Data was routinely collected at beam intensities of $1.5 \times 10^{7}$ particles $/ \mathrm{s}$.

In the offline analysis we were able to cleanly distinguish events produced in the target from those originating in the absorber. The dimuon mass resolution achieved was adequatre to reduce contamination of the continuum region above $4.0 \mathrm{GeV} / c^{2}$ by the $\psi^{\prime}$ resonance to $2.5 \pm 1 \%$. The data have been used to study $\psi$ production mechanisms and the validity of the DrellYan picture of high energy muon pair production [10-12].

\section{Acknowledgments}

The spectrometer was constructed and operated in the High Intensity Laboratory of Fermi National Accelerator Laboratory with support provided by the US Department of Energy, the International Programs and High Energy Physics Divisions of the US National
Science Foundation, the Natural Science and Engineering Research Council of Canada, the Quebec Department of Education, and the Hellanic Science and Technology Agency. We would like to thank the staffs of the various University Physics Departments, the Fermilab Physics Department, particularly C. Kerns, C. Lindenmeyer, and D. Tousignant, and the Fermilab Research Division for their support in this work.

\section{References}

[1] B. Cox, Fermilab Report $79 / 1$ (1979).

[2] The part of the Cherenkov counter which contained the mirror and the phototube housing was built by Princeton University.

[3] C. Kerns, IEEE Trans. Nucl. Sci. NS-24 (1977) 353.

[4] R. Kephardt and C. Kerns, Fermilab TM-1157 (1979).

[5] A. Van Ginneken and M. Awschalon, High Energy Particle Interactions in Large Targets, vol. 1, Fermilab.

[6] H. Areti et al., Nucl. Instr. and Meth. 212 (1983) 135.

[7] J. Biel and R.J. Dosen, Fermilab PN-112 (1979) and PN-148 (1981).

[8] C. Akerlof and D. Nitz, Proc. Digital Equipment Users Society, San Diego (1980) p. 801.

[9] J. Branson et al., Phys. Rev. Lett. 38 (1977) 1334 and ref. therein.

[10] E. Anassontzis et al., Contribution to the XXI Int. Conf. on High Energy Physics, Paris (1982) and Fermilab-Conf82/50-EXP.

[11] E. Anassontzis et al., Contribution to the XXI Int. Conf. on High Energy Physics, Paris (1982) and Fermilab-Conf82/49-EXP.

[12] E. Anassontzis et al., Fermilab-Pub-85/34, submitted to Phys. Rev. Lett. 